\title{
Clinical sequelae of cardiac device-related endocarditis
}

Cardiac device-related infective endocarditis (IE) is associated with a high prevalence of concomitant valve complications, and with high in-hospital and 1-year mortality. Removal of the device can improve the patient's chance of survival. These findings from the International Collaboration on Endocarditis-Prospective Cohort Study (ICE-PCS) have been reported in the Journal of the American Medical Association.

The use of implantable cardiac devices, such as pacemarkers and cardioverterdefibrillators, has increased dramatically over the past 20 years. This development has been accompanied by a rise in the prevalence of device-related infections, including IE. Despite the considerable burden that such complications place on health-care systems, "there are few studies evaluating the clinical characteristics, treatment, and outcome of devicerelated endocarditis," explains ICE-PCS investigator Professor Andrew Wang from Duke University Medical Center (Durham, NC, USA).
44 ...device removal, in addition to antibiotic therapy, should strongly be considered ... 77

The ICE-PCS registry operated between 2000 and 2006. During this time, 3,284 patients from 61 centers in 28 countries worldwide were enrolled. IE was diagnosed in 2,760 individuals, 177 (6.4\%) of whom had device-related IE. The majority of these patients were elderly men (median age 71.2 years). A substantial proportion also had diabetes mellitus. Staphylococcus aureus was the most common cause of infection. Moreover, in almost half of patients, device-related endocarditis was caused by a health-care-associated infection.

Concomitant valve disease, most commonly involving the tricuspid valve, was reported in 66 patients (37.3\%). Valve infection was associated with increased mortality, irrespective of whether or not the device was removed. In-hospital and 1 -year mortality was $14.7 \%$ and $23.2 \%$, respectively. Removal of the device and lead was associated with improved survival at 1 year (HR 0.42, 95\% CI 0.22-0.82, $P=0.01)$. However, removing the device had no affect on in-hospital mortality. The investigators speculate that this lack of benefit could relate to the high operative risk in these elderly patients.

"The results of this study imply that device removal, in addition to antibiotic therapy, should strongly be considered for all patients with cardiac device endocarditis," concludes Professor Wang. This viewpoint mirrors the recommendations of the AHA, which strongly supports device and lead removal in such cases. A need also exists for improved preventative measures, such as skin decontamination, and monitoring of patients to avoid progression to valve infection.

\section{Alexandra King}

Original article Athan, E. et al. Clinical characteristics and outcome of infective endocarditis involving implantable cardiac devices. JAMA 307, 1727-1735 (2012) 